\title{
The phenotypic features of osteogenesis imperfecta resulting from a mutation of the carboxyl-terminal pro $\alpha 1$ (I) propeptide that impairs the assembly of type I procollagen and formation of the extracellular matrix
}

Research Institute, The Hospital for Sick Children, 555 University Avenue, Toronto, Ontario, Canada M5G 1 X8 W G Cole

Department of Pathology, Royal Children's Hospital, Flemington Road, Parkville, Victoria 3052, Australia C W Chow

Department of Paediatrics, University of Melbourne, Royal Children's Hospital, Parkville, Victoria 3052, Australia J F Bateman

Department of Genetics, The Children's Hospital Camperdown New South Wales 2050, Australia

D O Sillence

Correspondence to: Professor Cole.

Received 30 November 1995 Revised version accepted for publication 29 May 1996

\author{
W G Cole, C W Chow, J F Bateman, D O Sillence
}

\begin{abstract}
The features of a baby with lethal perinatal osteogenesis imperfecta (OI-II), resulting from the substitution of tryptophan 94 by cysteine in the carboxyl-terminal propeptide of proal(I) chains of type I procollagen, were studied. The limbs and torso were of normal length, shape, and proportion. Similarly, all bones were of relatively normal shape and the long bones showed normal metaphyseal modelling. These clinical and radiographic features were similar to those observed in another baby with OI-II resulting from a mutation of the carboxy-terminal propeptide of pro $\alpha 1$ (I) chains but dissimilar from those reported in babies with OI-II resulting from helical mutations of type I collagen. (f Med Genet 1996;33:965-967)
\end{abstract}

Key words: osteogenesis imperfecta; procollagen; proxl(I) propeptide.

Osteogenesis imperfecta (OI) is a brittle bone disease that usually results from mutations in the COL1A1 or COL1A2 genes that encode the proal(I) and proa 2 (I) chains of type I procollagen, respectively. ${ }^{1}$ Most of the mutations involve the main triple helical domain and result in a dominant negative effect or in haploinsufficiency. ${ }^{12}$ Mutations of the carboxyl-terminal propeptides are rare but their consequences confirm the critical roles of this domain in the chain selection, registration, alignment, and disulphide bonding steps that are required for the assembly of type I procollagen trimers containing two pro $\alpha 1$ (I) chains and one pro $\alpha 2(\mathrm{I})$ chain..$^{3-8}$ The carboxyl-terminal propeptides are also important in the initiation of triple helix formation.

The severity of OI resulting from mutations of the carboxyl-terminal propeptides is dependent on the extent to which the mutations impair procollagen assembly. Two frameshift mutations, one in proal(I) and the other in proa2(I), completely prevented the incorporation of the mutant chains into type I procollagen trimers resulting in haploinsufficiency. ${ }^{34}$ Heterozygotes for the COL1A1 mutation had mild OI type I while heterozygotes for the COL1A2 mutation were clinically normal. Homozygotes for the latter mutation had moderately severe OI type III. ${ }^{5}$ In contrast, five different carboxyl-terminal propeptide mutations, which impaired but did not prevent the assembly of type I procollagen trimeric molecules, resulted in lethal OI. ${ }^{6-8}$ This report concerns one of the latter babies (OI64) who was heterozygous for a single base change in exon 49 of COL1A1 that converted the codon for the proal(I) carboxyl-terminal propeptide residue 94 from tryptophan (TGG) to cysteine (TGT) ${ }^{8}$ The mutation disturbed procollagen folding and retarded the formation of disulphide linked trimers. The endoplasmic reticulum resident molecular chaperone BiP, which binds to malfolded proteins, was induced and bound to type I procollagen in the OI64 fibroblasts. Unassembled mutant pro $\alpha 1(\mathrm{I})$ chains were also retained in the rough endoplasmic reticulum. Although most of the mutant type I procollagen molecules and unassembled mutant chains were degraded in the rough endoplasmic reticulum, some of the type I procollagen molecules containing mutant pro $\alpha 1$ (I) chains were secreted. These molecules were enzymatically overmodified owing to delayed formation of the triple helix. The overall effect of the mutation was to produce a major biosynthetic deficiency of type I procollagen that reduced the accumulation of type I collagen in the extracellular matrix to approximately $20 \%$ of normal. In this paper we describe the clinical, radiographical, and pathological features of this baby with lethal OI.

\section{Case report}

CLINICAL HISTORY

The proband (OI64) was born at 38 weeks' gestation with numerous fractures of the limbs, skull, and ribs and with subarachnoid and subdural haemorrhages. Death from respiratory distress occurred within hours of birth.

\section{RADIOGRAPHICAL FEATURES}

There was generalised osteopenia and numerous fractures of the long bones and skull (fig 1). The skull contained multiple wormian bones. However, the long bones were of relatively normal shape and showed normal metaphyseal modelling (fig 2). The shapes of the vertebrae, pelvis, and the bones of the hands and feet were also relatively normal. 


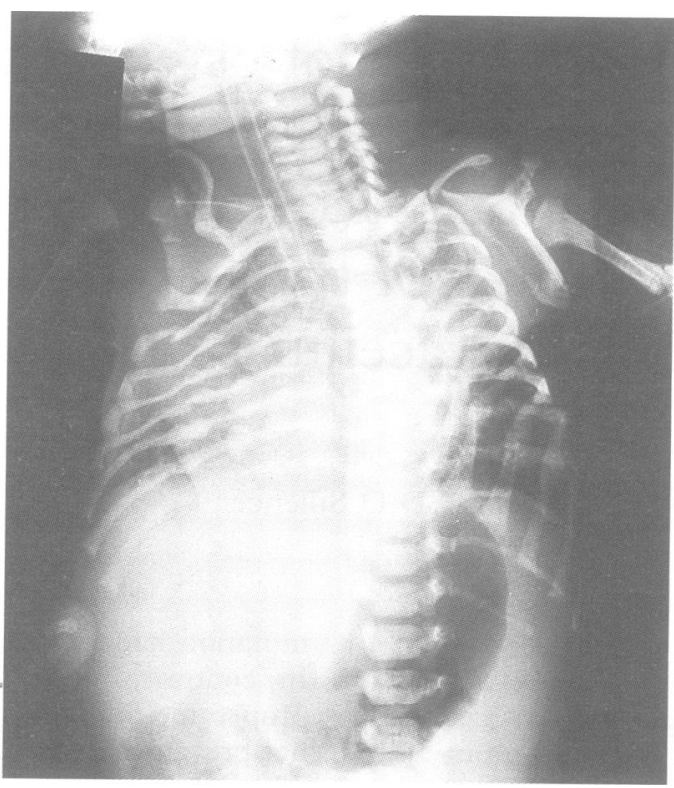

Figure 1 Anteroposterior radiograph of the torso. The ribs contain numerous fresh and healing fractures. The vertebrae are well formed.

\section{PATHOLOGICAL FEATURES}

The birth weight was $3060 \mathrm{~g}$ (50th centile) and the total body length was $49 \mathrm{~cm}$ (50th centile). The sclerae were deep blue. The limbs and torso were all of normal length, shape, and proportion. The skin, especially over the thighs, was lax. The joints were also lax and fractures were palpable in all long bones.

Necropsy showed fresh and healing fractures of all major long bones. There were healing nodules on all ribs, particularly posteriorly. In contrast, the pelvis and vertebrae were not fractured.

The head circumference was $35.5 \mathrm{~cm}$ (75th centile) and the very thin calvarium contained multiple fractures and wormian bones. There were no fractures of the base of the skull. There were extensive subdural and subarachnoid haemorrhages. The brain, which was of normal weight, appeared normal except where compressed by the haematomas. All other viscera were of normal appearance and size.

Histological examination showed normal growth plates in the long bones and ribs. However, the metaphyseal trabeculae contained persistent cartilaginous cores with very thin overlying osteoid seams (fig 3). The cartilaginous cores persisted into the diaphyseal bone. The bone matrix was intensely basophilic and the osteoblasts were plump and more numerous than normal. There was little bone matrix around each osteoblast. The metaphyseal and diaphyseal bone was woven with focal areas of lamellar bone (fig 4). There were no Haversian systems, although in some areas the osteoblasts produced pseudoHaversian systems. The vertebral bodies contained trabeculae of woven bone and near the vertebral end plates the trabeculae contained cartilaginous cores. The calvarium was composed of $0.4 \mathrm{~mm}$ thick plates of hypercellular woven bone which lacked a diploic space.

The sclerae were of normal thickness but the collagen fibres were less compact and more

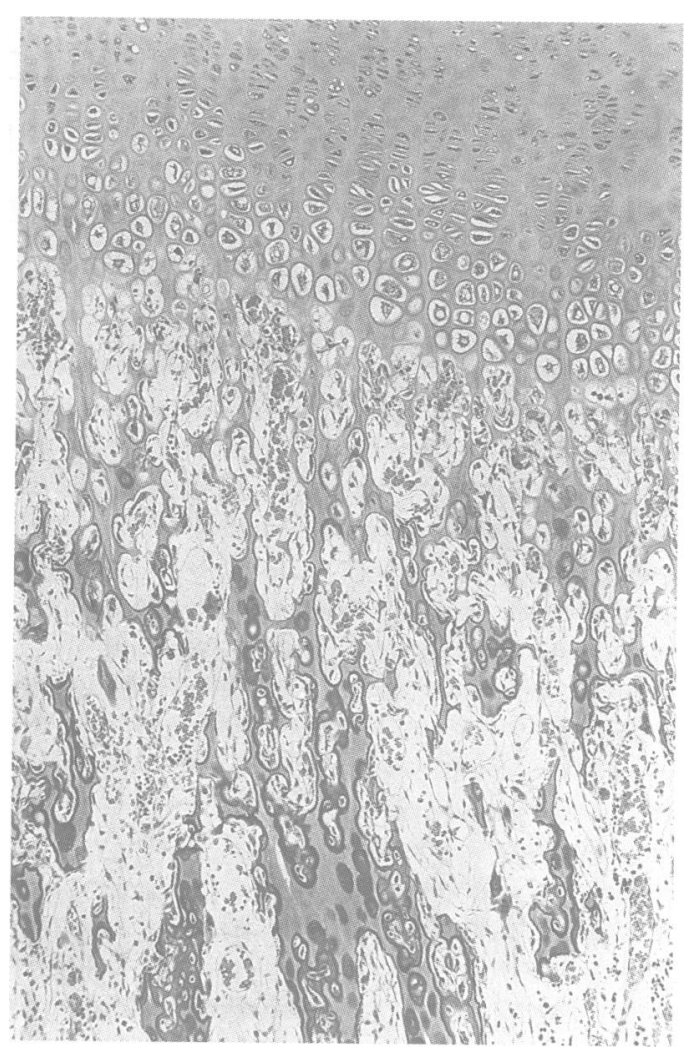

Figure 3 Light microscopy of the growth plate of the femur. The cartilaginous architecture is normal. The trabeculae are covered by a thin layer of osteoid and contain persistent cartilage cores.

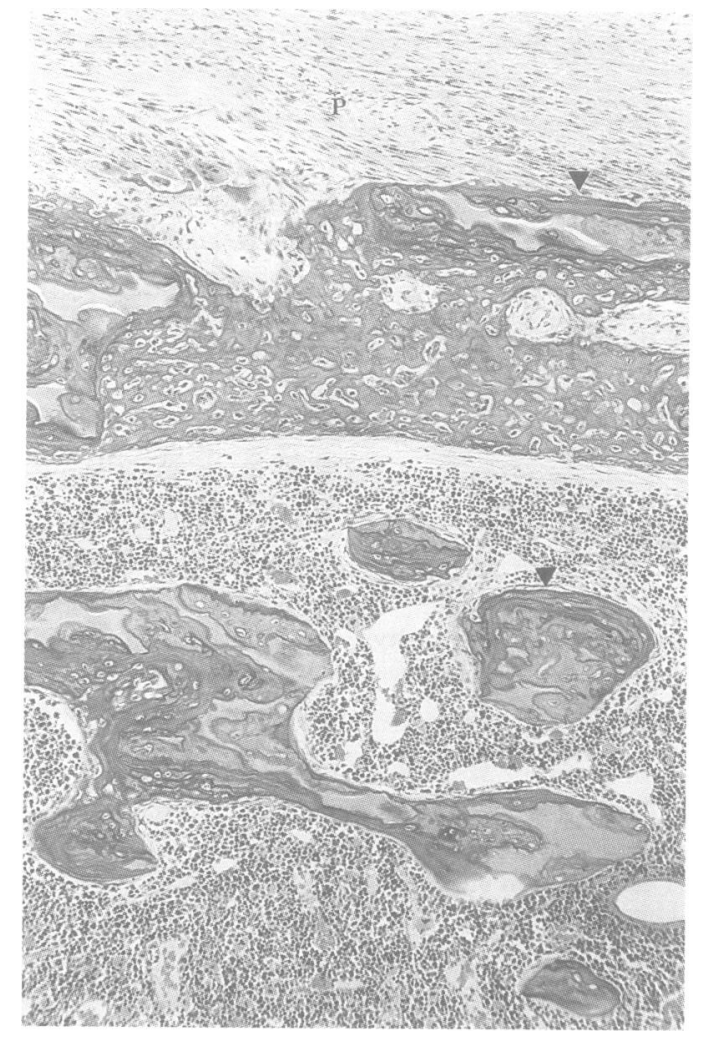

Figure 4 Light microscopy of woven cortical bone. The osteoblasts are plump and closely spaced. Cartilage cores persist in the cortical and cancellous bone. There are focal areas of lamellar bone (arrowheads). The periosteum is labelled $P$. 
randomly orientated than normal. The cornea had a loose pale inner zone. The dermis was thinner than normal.

Apart from haemorrhages, the brain and lungs were histologically normal. All other viscera were also histologically normal.

\section{Discussion}

Many of the radiographical, pathological, and biochemical features of the proband are similar to those of a baby (OI26) who had a lethal perinatal form of OI owing to a heterozygous frameshift mutation of the carboxyl-terminal propeptide of pro $\alpha 1$ (I) chains. ${ }^{79}$ The mutation produced a change in the amino acid sequence beginning at valine 106 of the carboxyl-terminal propeptide and resulted in a truncated chain. The propeptide lacked asparagine 147 , which normally carries an $\mathrm{N}$ linked oligosaccharide unit, and had an altered distribution of cysteine residues. The major clinical difference was that OI26 was born earlier at 35 weeks' gestation and was smaller, being below the 10 th centile for weight, length, and head circumference. It is likely, however, that the many similarities between OI64 and OI26 were because they shared a similar severe deficiency in the amount of type I collagen that was available to form the extracellular matrix. They also shared a similar maturational defect in osteogenesis in which the numerous plump osteoblasts and woven bone matrix maintained an appearance more typical of early embryonic bone. ${ }^{10}$

The relatively normal shapes of the torso, limbs, and bones in OI64 and OI26 were the main features that distinguished them from the more common forms of lethal perinatal OI. The latter forms of OI, which are the result of mutations in the helical domains of the type I collagen chains, have been classified into three groups. ${ }^{11}$ Group A have broad crumpled long bones and beaded ribs; group B have broad crumpled long bones with little beading of the ribs; and group $C$ have thin, fractured, cylindrical, dysplastic long bones and thin beaded ribs. We have designated the distinct clinical and radiological features of OI64 and OI26 as group D. In groups $A$ to $C$, the extracellular matrix contains a mixture of normal and mutant type I collagen molecules in contrast to the extracellular matrix of OI64 and OI26, group D, which contains a severely reduced amount of predominantly normal type I collagen molecules. However, the histological appearances of the bone matrix and osteoblasts in OI26 and OI64 were indistinguishable from those reported in groups $\mathrm{A}$ to $\mathrm{C} .^{2}$

Three additional cases of lethal osteogenesis imperfecta resulting from mutations of the carboxyl-terminal propeptide of proal(I) chains also shared the biochemical features of OI64 and OI26. A second trimester fetus (86146) who was stillborn had a heterozygous deletion of six base pairs that resulted in the deletion of glutamic acid 119 and tyrosine $120 .^{6}$ A term baby (86-237), who died at a day of age from respiratory failure, had a heterozygous substitution of histidine 59 by aspartic acid. ${ }^{6}$ Finally, a 17 week fetus (88-251) delivered spontaneously and was shown to be heterozygous for the substitution of arginine 170 by leucine. ${ }^{6}$

The mutations in these five cases resulted in severe reductions in the amount of type I collagen available for formation of the extracellular matrix and some of the collagen incorporated into the matrix was enzymatically overmodified. Increased levels of intracellular degradation of mutant collagen molecules and monomeric pro $\alpha$ chains were the major factors that produced the severe deficiency in secreted type I procollagen. The endoplasmic reticulum mediated quality control of type I collagen production in these cases was in large part because of the increased production of the endoplasmic reticulum resident binding protein, BiP, which binds to malformed proteins. ${ }^{712}$ It specifically bound to type I procollagen produced by the OI fibroblasts but not control cells. This quality control mechanism was not stimulated in the more common cases of OI resulting from mutations in the triple helical domains of the type I collagen chains. ${ }^{12}$ These findings highlighted the importance of the carboxyl-terminal propeptide in normal type I collagen biosynthesis and in the pathogenesis of a subset of cases of lethal OI.

This work was supported by grants from the Medical Research Council of Canada (WGC), The Research Institute of The Hospital for Sick Children, Toronto, and the NH \& MRC of Australia (JFB). We wish to thank P Bale for necropsy details and $\mathrm{P}$ Byers for his previous contribution to the biochemical characterisation of OI-64.

1 Byers PH, Wallis GA, Willing MC. Osteogenesis imperfecta: translation of mutation to phenotype. $\mathscr{f}$ Med Genet 1991; 28:433-42.

2 Cole WG, Dalgleish R. Perinatal lethal osteogenesis imperfecta. F Med Genet 1995;32:284-9.

3 Willing MC, Cohn DH, Byers PH. Frameshift mutation near the $3^{\prime}$ end of the COLlAl gene of type I collagen predicts an elongated pro alpha $1(\mathrm{I})$ chain and results in osteogenesis imperfecta type I. $\mathcal{f}$ Clin Invest 1990;85: 282-90.

4 Pihlajaniemi T, Dickson LLA, Pope FM, et al. Osteogenesis imperfecta: cloning of a pro-alpha 2(I) collagen gene with imperfecta: cloning of a pro-alpha 2(1) collagen gene with

5 Nicholls AC, Osse G, Schloon HG, et al. The clinical features of homozygous alpha 2(I) collagen deficient osfeatures of homozygous alpha 2(I) collagen deficient

6 Chessler SD, Wallis GA, Byers PH. Mutations in the carboxyl-terminal propeptide of the pro alpha 1 (I) chain of type I collagen result in defective chain association and produce lethal osteogenesis imperfecta. F Biol Chem 1993; 268:18218-25.

7 Bateman JF, Lamande SR, Dahl HHM, Chan D, Mascara T, Cole WG. A frameshift mutation results in a truncated nonfunctional carboxy-terminal pro $\alpha 1$ (I) propeptide of type I collagen in osteogenesis imperfecta. $7 \mathrm{Biol}$ Chem 1989;264:10960-4

8 Lamande SR, Chessler SD, Golub SB, et al. Endoplasmic reticulum-mediated quality control of type I collagen producticulum uction by cells from carboxyl-terminal propeptide which impair subur 1995;270:8642-9.

9 Cole WG, Campbell PE, Rogers JG, Bateman JF. The clinical features of osteogenesis imperfecta resulting from a non-functional carboxy-terminal pro $\alpha 1$ (I) propeptide of type I procollagen and a severe deficiency of normal type I collagen in tissues. $\mathcal{F}$ Med Genet 1990;27:545-51.

10 Aubin JE, Turksen K, Heersche JNM. In: Noda M, ed. Osteoblast cell lineage in cellular and molecular biology of bone. San Diego: Academic Press, 1993:1-45.

11 Sillence DO, Senn A, Danks DM. Genetic heterogeneity in osteogenesis imperfecta. F Med Genet 1979;16:101-16.

12 Chessler SD, Byers PH. BiP binds type-I procollagen pro alpha chains with mutations in the carboxyl-terminal propeptide synthesized by cells from patients with osteogenesis imperfecta. F Biol Chem 1993;268:18226-33. 